\title{
A JET MODEL OF HERBIG-HARO OBJECTS
}

\author{
M.J. Wilson \\ Department of Applied Mathematics \\ The University \\ Leeds LS2 9JT \\ S.A.E.G. Falle and D.E. Innes \\ Max-Planck-Institüt für Kernphysik \\ Postfach 1039 80, \\ D-6900 Heidelberg
}

\begin{abstract}
We present results of steady jet calculations in which the cooling and compression behind internal shocks leads to optical emission with an intensity pattern similar to the regular well-aligned emission knots characteristic of stellar jets.
\end{abstract}

There are, at present, known to be a number of well collimated 'jet-like' features associated with young stars or IR sources (Mundt \& Fried 1983; Reipurth et al. 1986; Mundt et al. 1986). Many of these jets show knots of enhanced optical emission with spectra typical of low-excitation shock-heated gas (Mundt et al. 1986), a good example being the jet associated with HH-34 (Reipurth et al. 1986; Bührke \& Mundt 1987).

It is well known (e.g. Prandtl 1952) that shocks are readily excited in a steady supersonic gas jet if it is initially out of pressure balance with its surroundings. Sanders (1983) proposed a sufficient condition for a non-uniform external pressure to produce shocks in a jet, while Falle \& Wilson (1985) and Wilson \& Falle (1985) derived a necessary and sufficient condition. This work indicates that the chances of shocks being induced in stellar jets by a pressure mismatch to its surroundings are very strong indeed.

Our assumptions are that the jet is axisymmetric, supersonic and steady (this being valid if the time-scale for changes in the external medium is greater than the flow-time down the jet). We can then use a Godunov scheme for steady supersonic flow (Glaz \& Wardlaw 1985) to model the flow within the jet. The numerical scheme includes the dynamical effects due to radiative cooling and allows us to calculate the emission from any shock-heated material within the jet.

In this paper we present a calculation representative of our results to date. Shown in Figure 1 are contours of pressure for a jet which experiences an external pressure variation sufficient to excite a series of incident and reflected shock pairs, very much like those found in a perturbed adiabatic jet (e.g. Falle \& Wilson 1985). The effect of the cooling is to increase the compression behind the shocks which, as can be seen, leads to a decrease in jet radius and shock strength. 


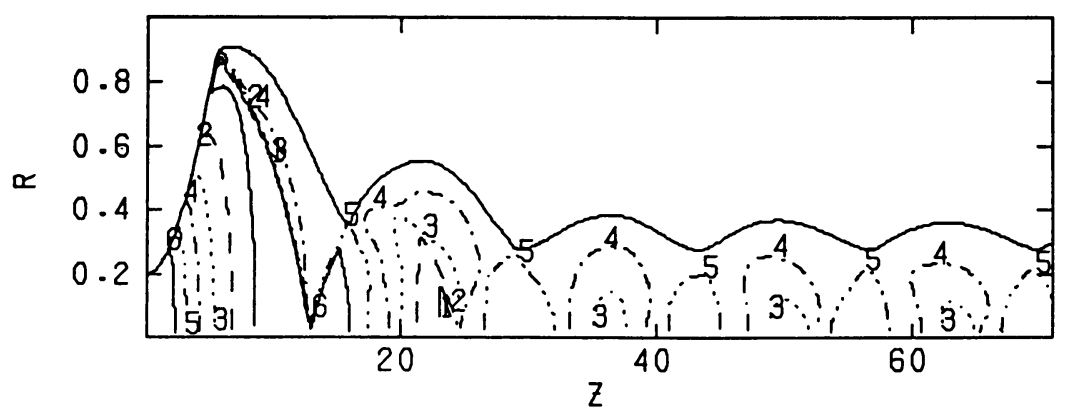

Figure 1. Log pressure contours for a steady jet with radius $R$ and length $Z$ (scaled units). The pressure increases from contour 1 to 6 with contour spacing $\log P=0.38$.

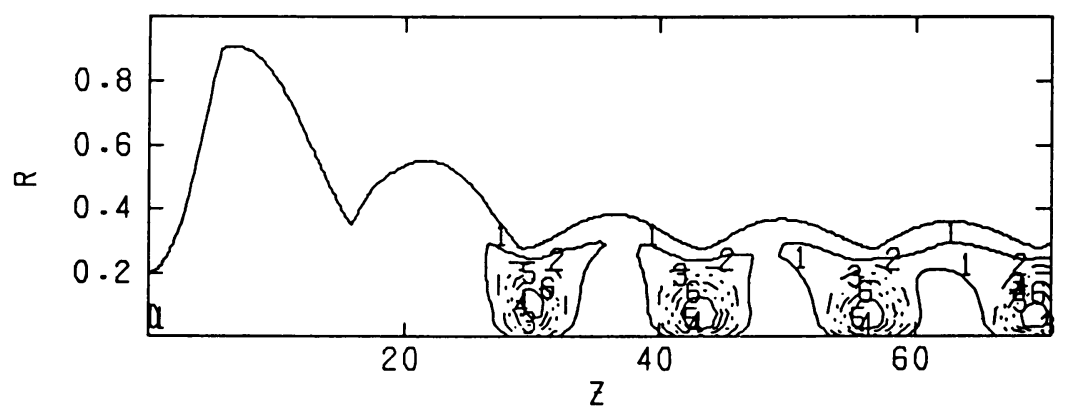

Figure 2. Linear [O I] $\lambda \lambda 6302,6365$ intensity contours for a line-of-sight perpendicular to the jet axis.

The integrated emission from the jet in the [O I] $\lambda \lambda 6302,6365$ line is shown in Figure 2. One finds that the optical emission from the jet is concentrated in a series of bright knots whose spacing is approximately $2 M \times R$, where $M$ is the jet mach number and $R$ is the jet radius. Each knot is associated with the high density region behind a reflected shock. The density enhancement in an element of fluid having passed through an incident/reflected shock pair can be up to a factor of 100 depending upon the cooling length. Using the velocity structure and emissivity of the gas, line profile maps along the jet can be calculated for the optical forbidden lines. We find that those regions with the lowest velocity dispersion correspond to the bright knots.

\section{References}

Bührke, T. \& Mundt, R, 1987, this volume.

Falle, S.A.E.G. \& Wilson, M.J., 1985, Mon. Not. Roy. Astr. Soc., 216, 79.

Glaz, H.M. \& Wardlaw, A.B., 1985, J. Comput. Phys., 58, 157.

Mundt, R., Brugel, E.W. \& Bührke, T, 1986, preprint.

Mundt, R. \& Fried, J.W., 1983, Astrophys. J. Letters, 274, L83.

Prandtl, L., 1952, Fluid Dynamics, (Blackie: London).

Reipurth, B., Bally, J., Graham, J.A., Lane, A. \& Zealey, W.J., 1986, Astron. \& Astrophys., in press.

Sanders, R.H., 1983, Astrophys. J., 266, 73.

Wilson, M.J. \& Falle, S.A.E.G., 1985, Mon. Not. Roy. Astr. Soc., 216, 971. 